\title{
Avaliação da produção de protease utilizando planejamento fatorial por Streptomyces parvulus DPUA 1573
}

\author{
J. M. SILVA ${ }^{1}$, T. S. PORTO², A.L.F. PORTO ${ }^{1}$ e C.S. PORTO ${ }^{1}$ \\ ${ }^{1}$ Universidade Federal Rural de Pernambuco, Departamento de Morfologia e Fisiologia Animal \\ (DMFA) \\ ${ }^{2}$ Universidade Federal Rural de Pernambuco, Unidade de Garanhuns (UAG) \\ E-mail para contato: milasporto@yahoo.com.br
}

\begin{abstract}
RESUMO - O objetivo deste trabalho foi selecionar as condições mais adequadas para a produção de protease utilizando planejamento fatorial pelo Streptomyces parvulus DPUA 1573. As variáveis de produção (concentrações da fonte de carbono- $0 \mathrm{~g} / \mathrm{L} ; 0,5 \mathrm{~g} / \mathrm{L} ; 1,0$ g/L, de nitrogênio- $0,5 \% ; 1,0 \% ; 1,5 \%$; agitação- 150,200 e $250 \mathrm{rpm}$; temperatura- 28, 32 e $37^{\circ} \mathrm{C}$ ) foram determinadas e analisadas por um planejamento fatorial $2^{4}$ tendo como variável resposta produção de protease e biomassa celular. Foi verificado que as variáveis independentes que mais influenciaram na produção foram a temperatura e agitação, sendo o ensaio 3 selecionado $\left(0,5 \%\right.$ de farinha de soja, $1,0 \mathrm{~g} / \mathrm{L}$ de glicose em $150 \mathrm{rpm}$ a $\left.28^{\circ} \mathrm{C}\right)$ com $347 \mathrm{U} / \mathrm{mL}$. A maior atividade proteásica foi obtida em 48 horas. Logo, a bactéria filamentosa Streptomyces parvulus DPUA 1573, apresentou atividade proteásica significativa podendo ser inserida no mercado enzimático.
\end{abstract}

\section{INTRODUÇÃO}

As proteases são enzimas diversificadas, representam um dos maiores grupos de enzimas industriais. A sua função é de hidrolisar ligações peptídicas para fins industriais, apresentam fácil capacidade de manuseio e mais rentável em razão de suas diferentes aplicações nas indústrias biotecnológicas, sendo um fator que facilita o fomento às pesquisas científicas com as proteases. (Li et al., 2013).

Estes tipos de enzimas apresentam diferentes fontes, no entanto as proteases microbianas se destacam no meio científico devido à sua capacidade metabólica celular ser considerada de baixo custo, facilitando no aumento de escala e no crescimento em um curto espaço de tempo, sem depender da sazonalidade produtora. Muitos micro-organismos produzem estas proteases, porém as bactérias filamentosas do gênero Streptomyces merece destaque por serem conhecidas cientificamente pelo seu potencial na produção de biocompostos de interesse industrial, como antibióticos, ácido clavulânico e enzimas (Awad et al., 2013; Dubey e Prasad et al., 2014; Mostafa et al., 2012).

Portanto, esta pesquisa teve por objetivo analisar e selecionar os parâmetros mais adequados para obter maiores rendimentos na produção de protease de interesse industrial por Streptomyces 
parvulus. DPUA 1573 isolado de líquen da Região Amazônica, utilizando um planejamento estatístico.

\section{Material e Métodos}

\subsection{Micro-organismo}

A linhagem Streptomyces parvulus DPUA 1573 foi isolada de líquen da Região Amazônia. Para a sua reativação foi utilizado o meio de cultivo ISP2 (Pridham et al., 1957), na temperatura de $28^{\circ} \mathrm{C}$ a 200 rpm por 48 horas.

\subsection{Meio para produção proteases pelo Streptomyces parvulus DPUA 1573}

Para a produção foi utilizado o meio MS-2 (Porto et al., 1996), a autoclavagem do meio de cultivo foi a $121^{\circ} \mathrm{C}$, a 1 atm de pressão, durante 30 minutos. O crescimento celular e produção de protease foram analisados a cada 24 horas até 120 horas.

\subsection{Planejamento fatorial para a produção de protease por Streptomyces parvulus DPUA 1573}

Os estudos de produção foram realizados com um auxílio de um planejamento fatorial completo $2^{4}$ com 4 pontos centrais. Sendo como fatores avaliados, as concentrações de fontes de nitrogênio e de carbono, agitação e temperatura, conforme apresentado na Tabela 1.

Tabela 1. Níveis das variáveis do planejamento fatorial $2^{4}$ na produção de protease

\begin{tabular}{lccc}
\hline \multicolumn{1}{c}{ Variáveis } & \multicolumn{3}{c}{ Níveis } \\
\cline { 2 - 4 } & Menor $(-1)$ & Central & Maior $(+1)$ \\
\hline Farinha de soja $(\%)$ & 0,5 & 1 & 1,5 \\
Glicose $(\mathbf{g} / \mathbf{L})$ & 0 & 0,5 & 1 \\
Agitação $(\mathbf{r p m})$ & 150 & 200 & 250 \\
Temperatura $\left({ }^{\circ} \mathbf{C}\right)$ & 28 & 32 & 37 \\
\hline
\end{tabular}

\subsection{Determinação do crescimento celular}

O crescimento celular foi estudado pela densidade óptica das amostras ao comprimento de onda a $600 \mathrm{~nm}$, em espectrofotômetro (Bel Protonics- SP 2000 UV). Como também por peso seco, o conteúdo do frasco foi filtrado em papel filtro seco previamente pesado, em seguida o papel de filtro juntamente com a massa celular foi levado em micro-ondas na potência baixa por 10 minutos (Olsson e Nielsen, 1997). 


\subsection{Atividade proteásica}

A atividade proteásica foi analisada de acordo com a metodologia de Ginther, 1979 modificado, que consiste na mistura de azocaseína a $1 \%$ com $0,2 \mathrm{M}$ de TRIS-HCL no pH 7,2 juntamente com $200 \mu \mathrm{L}$ do líquido metabólito livres de células, os ensaios foram analisados por espectrofotometria na absorbância de $420 \mathrm{~nm}$.

\section{Resultados e Discussão}

$\mathrm{Na}$ Tabela 2 estão apresentados os valores da atividade proteásica do planejamento fatorial realizado no tempo de 48 horas, pode-se verificar que a produção de protease ocorreu em todas as condições do planejamento fatorial.

Das variáveis independentes estudadas (concentração das fontes de carbono e nitrogênio, temperatura e agitação), as que influenciaram significativamente a produção de protease foram a temperatura e agitação, visualizado no gráfico de Pareto (Figura 1). De acordo com a Figura 1, o aumento da temperatura e agitação tem efeito negativo nos valores de atividade proteásica, no entanto a interação dessas variáveis foi positiva, onde os valores de temperatura e agitação diminuindo conjuntamente aumenta a atividade proteásica, porém as variáveis independentes concentrações de nitrogênio e carbono não foram estatisticamente significativas: a agitação aumenta a taxa de oxigênio e a transferência de nutrientes para as células bacterianas como também resulta na degradação de proteases, devido à lise celular ou permeabilidade celular excessiva relacionada à abrasão por forças de cisalhamento e limitação de oxigênio; a temperatura afeta diretamente na síntese de proteases influenciando as reações bioquímicas (Rahman et al., 2005). Neste caso específico, a temperatura e a agitação foram os fatores determinantes para a causa da repressão na produção de protease, ou seja, quando comparados os ensaios 7, 11 e 15 com o ensaio 3, percebe-se que houve um maior valor em atividade proteásica $(347 \mathrm{U} / \mathrm{mL})$.

Nesta pesquisa, foram estudas três temperaturas $\left(28^{\circ} \mathrm{C} ; 32^{\circ} \mathrm{C} ; 37^{\circ} \mathrm{C}\right)$ e três valores de agitação (150 rpm; $200 \mathrm{rpm} ; 250 \mathrm{rpm}$ ), o maior grau de atividade proteolítica foi em $28^{\circ} \mathrm{C}$ e a agitação de 150 rpm, resultado este oposto ao relatado por Bajaj et al. (2011) cultivou a linhagem Streptomyces sp. DP2 na temperatura de $45^{\circ} \mathrm{C}$ em $250 \mathrm{rpm}$ para produção de protease, tendo valores inferiores em comparação a este trabalho. Similar ao observado na pesquisa de El-Shafei et al. (2010) trabalhando com a bactéria Streptomyces albidoflavus na produção de protease alcalina em $37^{\circ} \mathrm{C}$ a $170 \mathrm{rpm}$. 
Tabela 2. Resultados da produção de protease por Streptomyces parvulus DPUA 1573 com 48 horas de fermentação

\begin{tabular}{llllll}
\hline Ensaio & Soja $(\boldsymbol{\%})$ & Glicose $(\mathbf{g} / \mathbf{L})$ & Temperatura $\left({ }^{\circ} \mathbf{C}\right)$ & Agitação $(\mathbf{r p m})$ & $\mathbf{U} / \mathbf{m L}$ \\
\hline $\mathbf{1}$ & 0,5 & 0 & 28 & 150 & 174 \\
$\mathbf{2}$ & 1,5 & 0 & 28 & 150 & 180,6 \\
$\mathbf{3}$ & 0,5 & 1,0 & 28 & 150 & 347 \\
$\mathbf{4}$ & 1,5 & 1,0 & 28 & 150 & 65,6 \\
$\mathbf{5}$ & 0,5 & 0 & 37 & 150 & 37,3 \\
$\mathbf{6}$ & 1,5 & 0 & 37 & 150 & 69,3 \\
$\mathbf{7}$ & 0,5 & 1,0 & 37 & 150 & 10,3 \\
$\mathbf{8}$ & 1,5 & 1,0 & 37 & 150 & 45,6 \\
$\mathbf{9}$ & 0,5 & 0 & 28 & 250 & 43,3 \\
$\mathbf{1 0}$ & 1,5 & 0 & 28 & 250 & 49,3 \\
$\mathbf{1 1}$ & 0,5 & 1,0 & 28 & 250 & 44,3 \\
$\mathbf{1 2}$ & 1,5 & 1,0 & 28 & 250 & 34,3 \\
$\mathbf{1 3}$ & 0,5 & 0 & 37 & 250 & 33,3 \\
$\mathbf{1 4}$ & 1,5 & 0 & 37 & 250 & 30 \\
$\mathbf{1 5}$ & 0,5 & 1,0 & 37 & 250 & 20,6 \\
$\mathbf{1 6}$ & 1,5 & 1,0 & 37 & 250 & 34,6 \\
$\mathbf{1 7} \mathbf{C}$ & 1,0 & 0,5 & 32 & 200 & 155,6 \\
$\mathbf{1 8} \mathbf{C}$ & 1,0 & 0,5 & 32 & 200 & 110,3 \\
$\mathbf{1 9} \mathbf{C}$ & 1,0 & 0,5 & 32 & 200 & 114,6 \\
$\mathbf{2 0} \mathbf{C}$ & 1,0 & 0,5 & 32 & 200 & \\
\hline
\end{tabular}

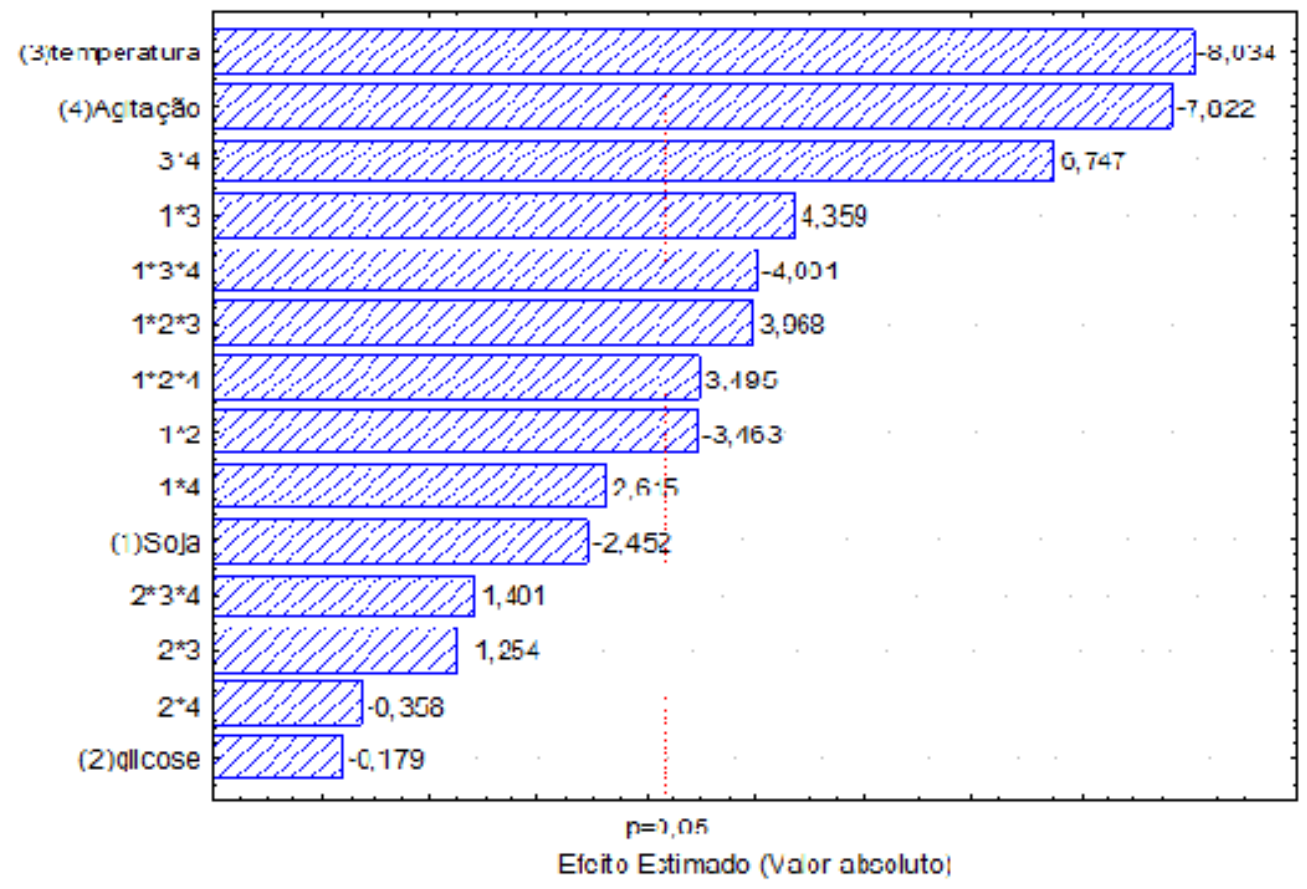


Figura 1 - Gráfico de Pareto dos efeitos das variáveis para produção de protease. (1) concentração de soja; (2) concentração de glicose; (3) temperatura; (4) agitação.

Em conformidade com o crescimento celular e ao tempo de produção, foi observado que em 24 horas de fermentação submersa ocorreu um início da produção de protease. De acordo com os resultados apresentado na Figura 2, a maior disponibilidade de nutrientes nas primeiras horas de cultivo deve-se pela presença de um pré-inóculo que reativa o micro-organismo de interesse, possibilitando assim, obter o maior valor de biomassa no menor tempo. O tempo de produção que apresentou os melhores resultados foi com 48 horas de crescimento; após este período foi verificado o continuo decréscimo de atividade até 120 horas de fermentação e também o crescimento microbiano.

Lazim et al, (2009) estudando a linhagem Streptomyces sp isolada de solo tunisiano, obteve o melhor tempo de produção com 72 horas de crescimento e Yuratmoko et al. (2007), que trabalhou com linhagens de Streptomyces sp, afirma que o início da produção ocorreu com 96 horas de fermentação e o seu pico foi com 168 horas. Pode ser verificado que pode haver diversidade de resultados apresentados na literatura com o mesmo gênero, devido a diferença do metabolismo dos Streptomyces relatados, em relação ao pico de produção variando de 48 horas a 168 horas, pela influência de diferentes condições de cultivo, tipo e concentração de substrato, temperatura, agitação, $\mathrm{pH}$.

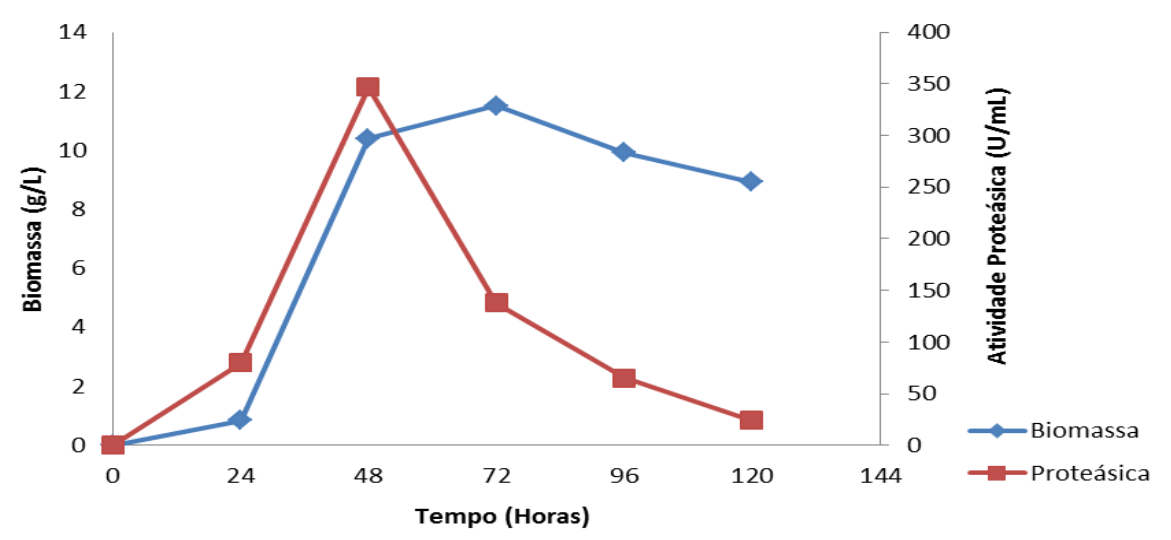

Figura 2 - Gráfico representando o crescimento microbiano da bactéria Streptomyces parvulus DPUA 1573 e sua produção de protease nas condições $0,5 \%$ de farinha de soja, $1 \mathrm{~g} / \mathrm{L}$ de glicose a $28^{\circ} \mathrm{C}$ em $150 \mathrm{rpm}$.

\section{CONCLUSÃO}

Com o resultados obtidos foi possível selecionar a condição mais adequada na produção de protease por Streptomyces parvulus DPUA 1573 com meio de produção instituído na concentração de $0,5 \%$ de farinha de soja (fonte de nitrogênio), $1 \mathrm{~g} / \mathrm{L}$ de glicose (fonte de carbono), a $150 \mathrm{rpm} \mathrm{em} 28^{\circ} \mathrm{C}$ 
no tempo de 48 horas de fermentação, com o valor de $347 \mathrm{U} / \mathrm{mL}$ de atividade proteásica, sendo favorável o tempo de produção e concentração de soja para a aplicação industrial.

\section{REFERÊNCIAS}

ARA, I.; BUKHARI, D.R.; WIJAYANTI, BAKIR, M.A.; V.N. Proteolytic activity of alkaliphilic, salt-tolerant actinomycetes from various regions in Saudi Arabia. African Journal of Biotechnology, v. 11(16), p. 3849-3857, 2012.

AWAD, H.M, MOSTAFA, E.E. SAAD, M.M.; SELIM, M.H.; HASSAN, H,M. Partial purification and characterization of extracellular protease from halophilic and thermotolerant strain Streptomyces pseudogrisiolus NC-15. Indian J. of Biochemistry e Biophysics, v 50, p. 305-311, 2013.

BAJAJ, B.K.; SHARMA, P. An alkali-thermotolerant extracellular protease from a newly isolated Streptomyces sp. DP2. New Biotechnology, v. 28(6), 2011.

EL-SHAFEI. H.; ABDEL-AZIZ, M.S.; GHALY, M.F, ABDALLA, A.A.H. Optimizing some factors affecting alkaline protease production by a marine bacterium Streptomyces albidoflavus. Proce. of fifth scient. envi. conf., 2010.

GINTHER, C.L. Sporulation and the- Production of Serine Protease and Cephamycin C by Streptomyces lactamdurans. Antim. agents and chem., v 15 (4), p. 522-526, 1979.

LAZIM, H.; MANKAI, H.; SLAMA, N.; BARKALLAH, I.; LIMAM, F. Production and optimization of thermophilic alkaline protease in solid-state fermentation by Streptomyces sp. CN902. J Ind Microbiol Biotechnol, v. 36, p. 531-537, 2009.

MOSTAFA, E.E.; MOATAZA, M.S.; AWAD, M.H.S.; HASSAN, H.M. Optimization conditions of extracellular proteases production from a Newly isolated Streptomyces pseudogrisiolus NCR - 15. EJournal of Chemistry, v 9(2), pp. 949-961, 2012.

OLSSON, L.; NIELSEN, J. On Line and in situ monitoring of biomass in submerged cultivations. Trends in Biotech., v. 15, p. 517-522, 1997.

PORTO, A.L.F.; CAMPOS-TAKAKI, G. M.; LIMA FILHO, J.L. Effects of culture conditions on protease production by Streptomyces clavuligerus growing soy bean flour médium. Ap. Biochem. Biotech.v.60, p.115-122., 1996.

PRIDHAM, T. G.; ADERSON, P.; FOLEY, C.; LINDENFELSER, L. A.; HESSELTINE, C. W.; BENEDICT, R. G. A. Selection of Media for Maintenance and Taxonomic Study of Streptomyces. Antibiot. M. p.947-953, 1957.

RAHMAN, R.N.Z.A.; GEOK, L.P.; BASRI, M.; SALLEH, A.B. Physical factors affecting the production of organic solvent-tolerant protease by Pseudomonas aeruginosa strain K. Bioresource Technology, v. 96, p. 429-436, 2005.

RASHMI, D.; PRASAD, R. Biophysical characterization of in vitro bound Streptomyces peucetius daunorubicin-serine protease complex. International J. of Biol. Macrom., v.64, pp. 111-114, 2014. 
VONOTHINI, G.; MURUGAN, M.; SIVAKUMAR, K.; SUDHA, S. Optimization of protease production by an actinomycete STRAI, PS-18A isolated from an estuarine shrimp pond. African J.l of Biotechnol, v. 7 (18), pp. 3225-3230, 2008.

YURATMOKO, D.; MUBARIK, N.R.; MERYANDIN, A. N. Screening of proteolytic enzymes of Streptomyces sp local strain and their characterization. Microbiol Indonesia, v 1, 2007. 